\title{
ARQUITETURA DE UM SISTEMA PARA MONITORAMENTO PROLONGADO DE SINAIS CLÍNICOS DA DOENÇA DE PARKINSON
}

\author{
A. P. Rodrigues ${ }^{1}$, M. F. Vieira ${ }^{1,2}$ e A.O. Andrade $^{3}$ \\ 1 Programa de Pós-Graduação em Engenharia Elétrica, Mecânica e de Computação, Universidade \\ Federal de Goiás, Goiânia, Brasil \\ ${ }^{2}$ Programa de Pós-Graduação em Ciências da Saúde, Universidade Federal de Goiás, Goiânia, \\ Brasil \\ ${ }^{3}$ Núcleo de Inovação e Avaliação Tecnológica em Saúde (NIATS), Programa de Pós-Graduação em \\ Engenharia Elétrica e Biomédica, Faculdade de Engenharia Elétrica, Universidade Federal de \\ Uberlândia, Uberlândia, Brasil \\ e-mail: adriano.pericles@gmail.com
}

\begin{abstract}
Resumo: A doença de Parkinson atinge cerca de $1 \%$ da população mundial acima de 65 anos e mesmo com várias pesquisas tentando mensurar ou identificar a doença, ainda há dificuldades em encontrar métodos e ferramentas que resultem em coletas de dados confiáveis devido a imprevisibilidade e variabilidade de seus sinais clínicos. Essa necessidade motivou a ideia de criar uma arquitetura de um sistema embarcado para o monitoramento com tempo prolongado de um indivíduo com a doença de Parkinson. O sistema deve se adequar a situações do dia a dia coletando dados inerciais e eletromiográficos com o mínimo de interferências possível, possuir uma taxa de amostragem alta e a capacidade de armazenamento desses dados em uma memória portátil para analises futuras.
\end{abstract}

Palavras-chave: Doença de Parkinson, Sensor inercial, Eletromiografia.

Abstract: Parkinson's disease affects about $1 \%$ of the
population over 65 years old and even with multiple
researches trying to measure or identify the disease,
there are still difficulties in finding methods and tools
that result in reliable data collection due to
unpredictability and variability of their clinical signs.
This necessity led to the idea of creating an architecture
of an embedded system for monitoring with extended
time of an individual with Parkinson's disease. The
system must adapt the situations of everyday life
collecting inertial and electromyography data with
minimal interference as possible, have a high sampling
rate and storage capacity of these data in a portable
memory for future analysis.

Keywords: Parkinson's disease, Inertial sensor, Electromyography.

\section{Introdução}

A doença de Parkinson (DP) se tornou uma das doenças neurológicas mais comuns da atualidade, atingindo, segundo a Organização Mundial da Saúde (OMS), cerca de $1 \%$ da população mundial acima de 65 anos. No Brasil estima-se que 200 mil pessoas sofram com a doença, que não possui cura até hoje [1]. Os cinco sinais clínicos mais comuns da DP são rigidez, acinesia (ausência de movimento), bradicinesia (lentidão de respostas físicas e psíquicas), tremor e instabilidade postural. Vários estudos estão sendo feitos para quantificar esses sintomas da DP a fim de identificar indivíduos com a doença o mais breve possível e com o avanço tecnológico, muitas pesquisas têm tido sucesso neste propósito, como o trabalho de Djuri-Jovii et al. [2], que implementaram um algoritmo para identificar se o indivíduo está parado ou oscilando, andando normal ou cambaleando, e para isso utilizou-se giroscópios no pé e na cintura, e acelerômetros no pé. Essas foram as características relevantes que apontam o grau de evolução da DP no trabalho feito.

Tien et al. [3] usaram um sensor inercial composto de giroscópios e acelerômetros colocados no pé do indivíduo para identificar anormalidades no padrão da marcha do sujeito com DP, e utilizando máquina de vetores de suporte para a avaliação de um classificador, obtendo taxas de sucesso de até $93 \%$ na discriminação entre indivíduos com e sem a DP.

Apesar do sucesso das pesquisas recentes, existem poucos estudos que levam em consideração a imprevisibilidade e variabilidade dos sinais clínicos da DP, sendo este o principal fator que nos motivou a propor a arquitetura de um sistema para monitoramento prolongado de pessoas com a DP.

Essa pesquisa propõe unificar tecnologias que ainda não foram integradas para criar um sistema embarcado preciso e portátil possibilitando o monitoramento em domicílio durante a execução de tarefas do dia a dia.

\section{Materiais e métodos}

Levando em consideração a proposta de um protocolo com tempo prolongado é possível perceber que variáveis externas serão impactantes na coleta dos dados pois interferências surgirão aleatoriamente, especialmente no sinal EMG, portanto foi escolhido 
nesta proposta de arquitetura uma nova ferramenta que substituirá o eletrodo de superfície convencional, sendo esse o sensor capacitivo PS25255 (Figura 1).

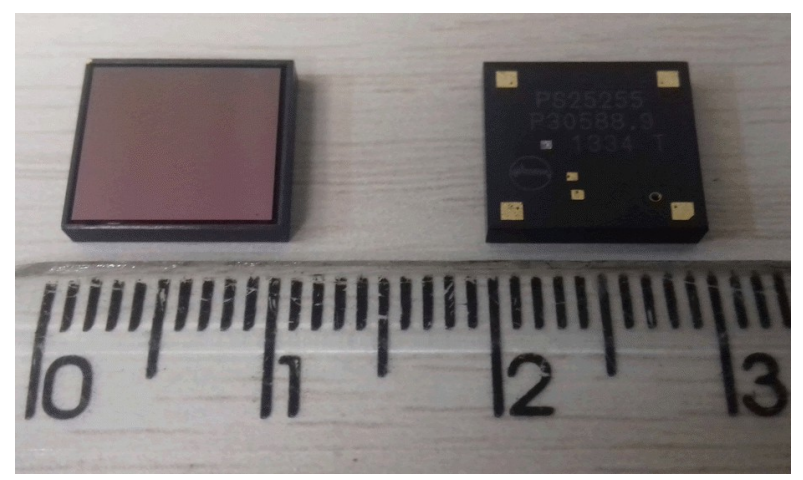

Figura 1: Sensor PS25255 frente e verso.

Este sensor elimina grande parte de interferências externas no sinal, funciona com baixa corrente e pode ser colocado diretamente sobre a pele seca ou roupas, tornando-o bem adequado para o monitoramento prolongado.

Outro aspecto importante do sistema será o uso de um condicionador de sinais biológicos na forma de um microchip (ADS1298) que possui taxa de amostragem variando de $250 \mathrm{~Hz}$ a $32 \mathrm{kHz}$, e amplificadores programáveis integrados [4]. O uso deste condicionador juntamente com o sensor capacitivo possibilitará o desenvolvimento de um sistema embarcado, de dimensões reduzidas, de modo a atendar os requisitos necessários ao monitoramento prolongado de indivíduos no lar.

Juntamente com a EMG é necessário coletar dados inerciais devido aos sintomas clínicos da DP, então foi selecionado o sensor MiniIMU-9 (Figura 2), que possui um giroscópio L3GD20, um acelerômetro LSM303DLHC e um magnetômetro, ambos de três eixos.

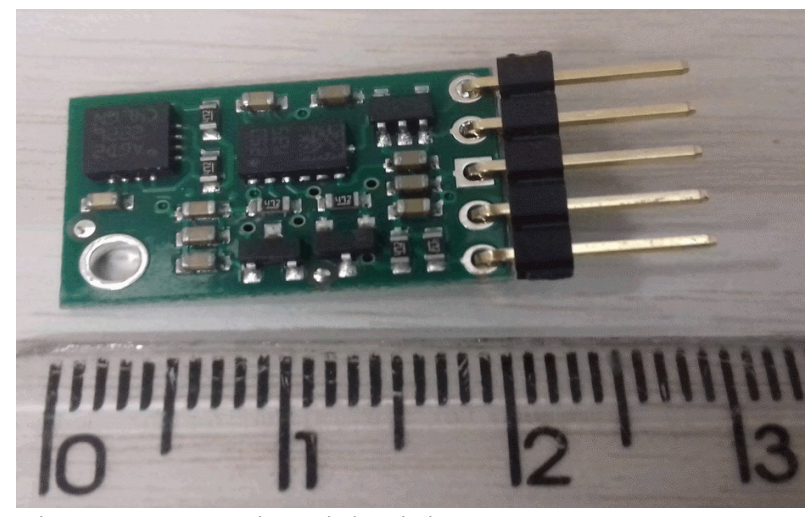

Figura 2: Sensor inercial MiniIMU-9.

Para fazer o registro de todas as informações será criada uma placa de circuito impresso com um circuito integrado que controlará o processo de coleta e armazenamento de dados.

\section{Resultados}

Como os sintomas da DP estão bastante presentes em membros periféricos, na postura e marcha, nossa proposta de arquitetura terá foco de avaliação de informações obtidas a partir dos punhos e cintura.

Nos punhos será necessário a coleta de dados de vários músculos, como por exemplo, os extensores e flexores do punho. Além disso, para quantificar os sinais clínicos da DP será necessária a coleta simultânea de dados inerciais e eletromiográficos.

Para a avaliação postural e da marcha será utilizado um cinto, que possui dois sensores inerciais que serão posicionados na região do quadril [5].

Conforme é possível observar na Figura 3, a proposta de arquitetura se mostra simples, porém a organização do sistema que será algo complexo.

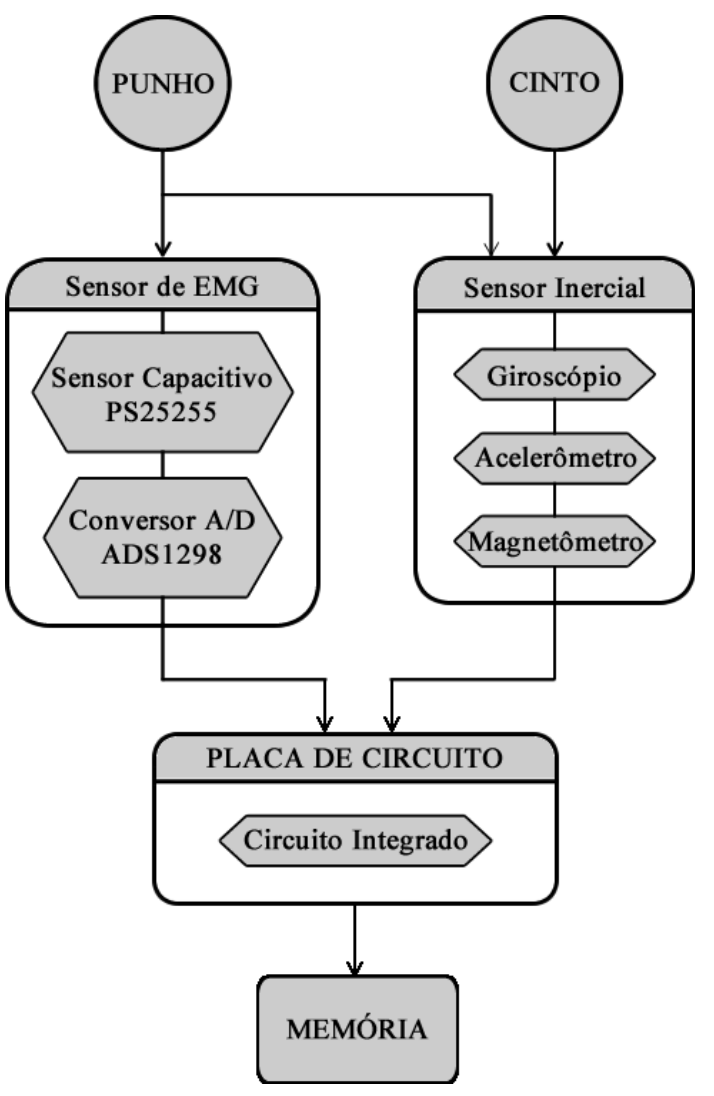

Figura 3: Arquitetura do sistema de monitoramento proposto.

\section{Conclusão}

A imprevisibilidade e variabilidade dos sinais clínicos da DP mostram que a arquitetura proposta seria bem adequada para gerar dados confiáveis para a classificação dos graus dos sintomas.

\section{Agradecimentos}

Agradecemos à CAPES, CNPq e FAPERGS pelo apoio financeiro e concessão de bolsas, ao Programa de 
Pós-Graduação em Engenharia Elétrica, Mecânica e de Computação da Universidade Federal de Goiás, ao Laboratório de Bioengenharia e Biomecânica da Universidade Federal de Goiás, ao Núcleo de Inovação e Avaliação Tecnológica em Saúde (NIATS) da Universidade Federal de Uberlândia.

\section{Referências}

[1] Conheça os sintomas do Mal de Parkinson [internet]. 2014 Out; Available from: http://www.brasil.gov.br/saude/2014/10/conheca-ossintomas-do-mal-de-parkinson.

[2] Djuri-Jovii M, Jovii NS, Milovanovi I, Radovanovi $\mathrm{S}$, Kresojevi N e Popovi MB. Classification of Walking Patterns in Parkinson's Disease Patients Based on Inertial Sensor Data. In: 10th Symposium on Neural Network Applications in Electrical Engineering; 2010 Set 23-25; Serbia; University of Belgrade.

[3] Tien I, Glaser SD, e Aminoff MJ. Characterization of Gait Abnormalities in Parkinson's Disease Using a Wireless Inertial Sensor System. In: 32nd Annual International Conference of the IEEE EMBS; 2010 agos e set 31-4; Buenos Aires; Argentina.

[4] Instruments TI. Low power consumption for biopotential measurement, 8-channel, 24-bit analog front-end. 2010 Jan. Available from: http://www.ti.com.

[5]Ferreira LCV. Análise da variabilidade da marcha de indivíduos com comprometimento articular do quadril por meio de sensores inerciais. Uberlândia: Universidade Federal de Uberlândia; 2016. 